International Journal of Computer Networks \& Communications (IJCNC) Vol.4, No.2, March 2012

\title{
RESOURCE OPTIMIZATION IN MOBILE COMMUNICATION NETWORKS WITH USER Profile-BASEd Algorithms
}

\author{
E. Martin and R. Bajcsy \\ Department of Electrical Engineering and Computer Science \\ University of California, Berkeley \\ California, USA \\ emartin@eecs.berkeley.edu
}

\begin{abstract}
In mobile communications networks, Location Management enables the roaming of the user in the coverage area. The employment of the call and mobility patterns of the user can help minimize the signaling costs involved in Location Management, and optimize the available radio resources. In this paper, we carry out an exhaustive analysis of the location update costs involved in a user profile-based Location Management algorithm, and compare its performance with the classical strategy of static location areas. As original contributions, we introduce two new algorithms to obtain the $\beta$ parameters, useful for the calculation of the Location Management signaling costs. Making use of these new algorithms, we show the convenience of the application of user profile-based strategies for Location Management in order to optimize the available radio resources, and we obtain practical guidelines for the optimum design of mobile communications networks.
\end{abstract}

\section{KEYWORDS}

Resource Management, Location Management, Location Update, Signaling Costs, Beta Parameters, Wireless Communications Networks, Algorithms.

\section{INTRODUCTION}

The recent growth in the number of users in mobile communications networks and the rise in the traffic generated by each user, are responsible for the increasing importance of Mobility Management in the access links to the networks. The proliferation of new protocols and algorithms aimed at enhancing the network capabilities and providing the user with more and better services has become a constant trend. Nevertheless, there are still open problems concerning Mobility Management that need to be tackled. Special attention must be paid to the efficient use of the scarce radio resources. Within Mobility Management, Location Management enables the roaming of the user in the coverage area, with the main tasks involved being location update and paging [1-6]. The location update procedure consists of informing the network about every new location the mobile terminal enters, while paging is employed by the network to deliver incoming calls to the user. The signaling messages involved in these two procedures consume a significant proportion of the available radio resources [7-10]. In order to minimize this signaling burden, the location area concept (a set of cells) is used, whereby the mobile terminal will inform the network about a change in its position only when the location area's border has been crossed. The employment of the call and mobility patterns of the user can help optimize the location area's dimensions and minimize signaling costs [11]. In fact, user profile-based algorithms for Location Management have proved to significantly reduce signaling costs [12-14]. In this type of algorithms, the most frequently visited location areas are

DOI : 10.5121/ijcnc.2012.4201 
assigned a probability coefficient consistent with the user's residence time in each one of them. Subsequently, the network creates a list to order the location areas according to those probabilities, and in the case of an incoming call, the location areas will be paged sequentially following their decreasing order of probability. When the mobile user exits the predetermined set of location areas, it will perform a location update operation in the first visited cell. Therefore, a profile in the form of a list is needed for each user, containing the identification of the most frequently visited location areas. In a simplified approach of this algorithm, only long term statistics (weeks or months) are memorized by the system, ignoring short term statistics (hours or days). And even this basic approach considering only long term statistics can bring important savings in location update operations. Recent examples making use of this approach can be found in reference [15], which describes an algorithm leveraging the user profile history to reduce location update costs, utilizing cascaded correlation neural networks trained on historical data of the user's movements, subsequently employed to predict the location of the user. In a similar way, reference [16] introduces a system to deliver personalized services to its customers based on the surrounding context and the user profile. Other researchers leverage this approach working with personalized queries, and using a degree of interest score to model user profiles [17-21].

In this paper, we carry out an exhaustive analysis of the location update costs involved in a user profile-based Location Management algorithm. We analyze the signaling costs in mobile communications networks with a two-tier architecture, making use of a typical user profilebased methodology, and compare its performance with the classical strategy of static location areas. As original contributions, we introduce two new algorithms to obtain the $\beta$ parameters, useful for the calculation of the Location Management signaling costs. Making use of these new algorithms, we show the convenience of the application of user profile-based algorithms for Location Management in order to optimize the available radio resources, and we obtain useful guidelines for the design of wireless communications networks.

The rest of this paper is organized as follows. In Section 2, we provide background information for the analysis of Location Management costs in mobile communications networks. In Section 3 , we introduce two novel algorithms to obtain the $\beta$ parameters used in the calculation of the location update costs for different Location Management strategies. Making use of these novel algorithms, Section 4 is devoted to the computation of the location update signaling costs for the classical strategy of fixed location areas, and Section 5 shows the computation of the location update costs for a typical user profile-based algorithm. Finally, the conclusions are drawn in Section 6.

\section{BACKGROUND INFORMATION FOR THE ANALYSIS OF LOCATION UPDATE COSTS}

In order to analyze the signaling burden related to a Location Management algorithm, a relationship between the call and mobility models of the user can be useful, as shown in [2229], where the call-to-mobility ratio is utilized. For the analysis that follows, we assume that the user moves randomly and that all the location areas under study have the same area, even if this size might not be optimum (dynamic location area size strategies are proposed in [30-35]). Under these assumptions, the frequency of the location updates depends on the speed of the mobile user, $v$, and the surface and perimeter length of the location areas. Taking into account that the location update operations can take place within a same VLR (case 1, with probability $\beta_{1}$ ), or between two VLRs, making use of the Temporary Mobile Subscriber Identity (case 2.1, with probability $\beta_{21}$ ), or making use of the International Mobile Subscriber Identity (case 2.2, 
International Journal of Computer Networks \& Communications (IJCNC) Vol.4, No.2, March 2012

with probability $\beta_{22}$ ), the location update costs for the classical strategy in mobile communications networks with a two-tier architecture can be expressed as follows [12-14]:

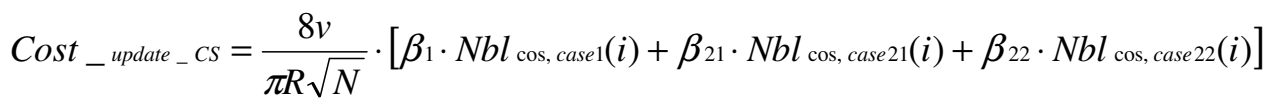

Where $R$ is the hexagonal cell side, $N$ is the number of cells per location area, and $N b l_{\text {cos, case }}(i)$ is the number of bytes generated by a location update at interface $i$ for any of the three different cases explained before. Defining a parameter called $\beta_{2}$ as the probability of location update using different VLRs, $\beta_{21}$ can be approximated by $80 \%$ of $\beta_{2}$ [36], and $\beta_{22}$ by $20 \%$ of $\beta_{2}$. In Section 3, we will introduce two new algorithms for the calculation of these parameters.

For a typical user profile-based algorithm, often called "Alternative Strategy (AS)" [12-13], the location update costs can be expressed as follows:

Cost_update $_{-} A S=\left(1-\sum_{i=1}^{k} \alpha_{i}\right) \cdot$ Cost $_{-}$update $C S$

Where $\alpha_{i}$ is the probability of finding a mobile user in the location area $a_{i}$, and $k$ is the number of location areas administered by this strategy.

\section{Calculation of the $\beta$ Parameters}

Assuming densely populated areas, with an average number of cells per location area of 10 [37], and an average number of location areas managed by a VLR of 5, the calculation of the $\beta$ parameters to obtain the location update costs will be tackled next.

Different algorithms can be used to obtain the values for the $\beta$ parameters. In this paper we analyze the cells in the network one by one and determine the probabilities of a mobile terminal with random movement getting into a new location area, whether within the same VLR or not, so that each cell is assigned a set of values, marked with a cross (denoted by " $\mathrm{x}$ ") or a dot (denoted by "•") in Figure 1, to reflect respectively the probabilities of crossing the location area border and moving outside the actual VLR administered zone or remaining within it.

The $\mathrm{x}$ and $\bullet$ numbers could be obtained through the mobile terminal's mobility parameters owned by the network operator, or through a geographical study of relative positions of the cells within the different location areas and the VLR administered zone itself. Considering this last option, the different numbers assigned to each cell can be made dependant upon the designer's criteria, for instance in the two following ways: first, if the designer just wants to reflect the fact that a cell is neighboring a different VLR administered zone/location area, or second, if the designer wants to reflect the exact proportionality between the number of neighboring cells from a different VLR administered zone and the number of neighboring cells from different location areas within the same VLR administered zone. These two alternatives lead to a couple of methods that we respectively name simple and advanced algorithms. 
International Journal of Computer Networks \& Communications (IJCNC) Vol.4, No.2, March 2012
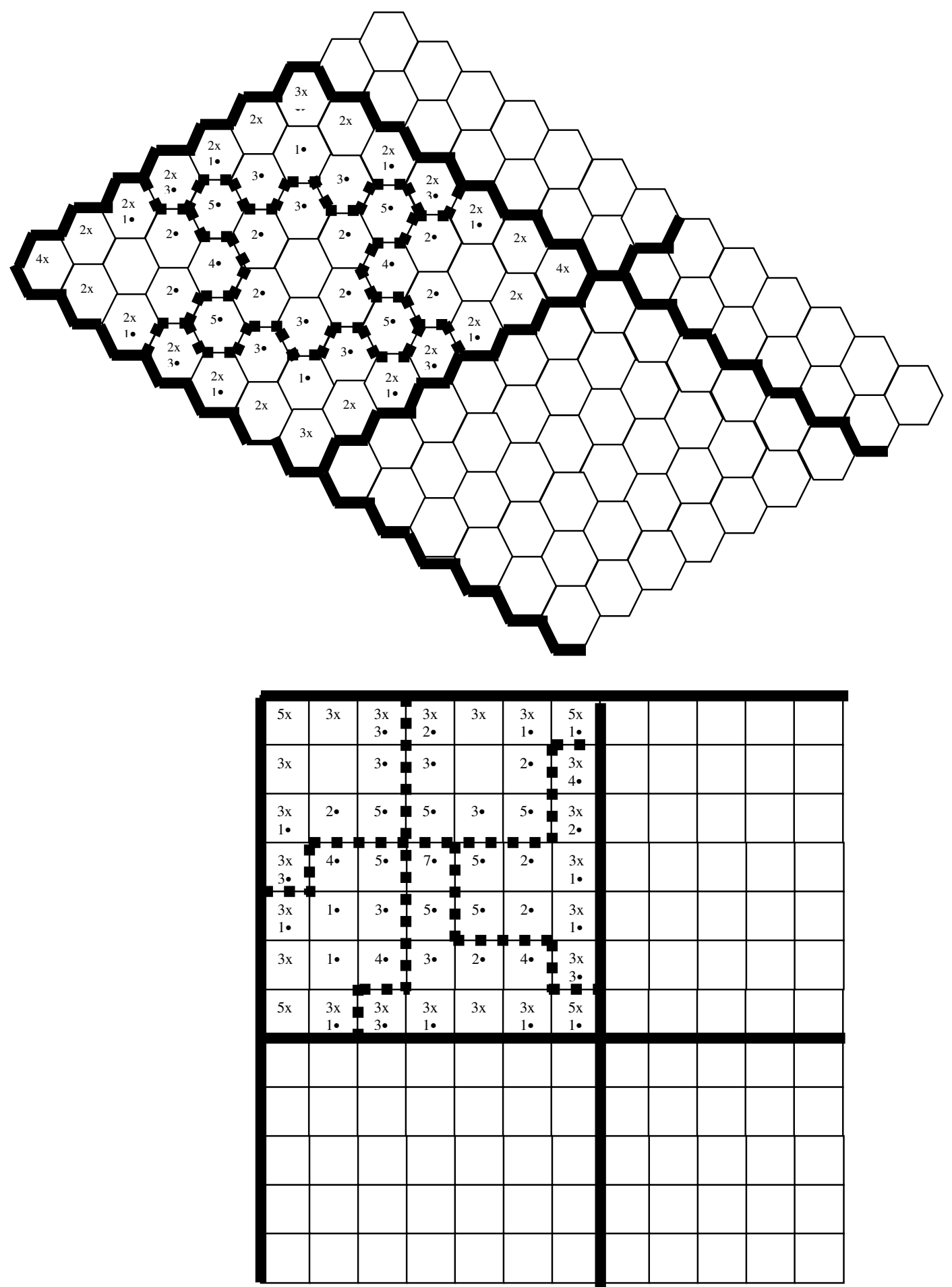

Geographical Area managed by a VLR $\quad$ x: possible crossing of L.A. border outside the

- - Location Area border VLR

Cell border within a Location Area

Figure 1. Calculation of basic parameters to obtain Location Management Costs, considering hexagonal or square shaped cells. 
International Journal of Computer Networks \& Communications (IJCNC) Vol.4, No.2, March 2012

\subsection{Simple Algorithm}

Taking for example a squared geographical area of dimensions $7 \cdot 7=49$ cells, so that the cells administered by a VLR can be grouped in 5 location areas with 10 cells each but one of them with 9, considering that every cell in the border of the VLR administered zone as a whole can be assigned an $\mathrm{x}$, and every cell sharing border with another location area within the same zone can be assigned a $\bullet$, the proportion between the number of $\bullet$ s and the sum of the number of xs and -s will represent the $\beta_{1}$ parameter, while the proportion between the number of xs and the sum of the number of xs and $\bullet$ s will represent the $\beta_{2}$ parameter. The results obtained for the referred deployment are: $\beta_{1}=40 /(40+24)=0.625$, and $\beta_{2}=24 /(40+24)=0.375$.

Considering now the same VLR administered area but with lower number of cells per location area $(9,7,6)$, so that the number of location areas increases to 6 , the results obtained are very similar: $\beta_{1}=41 /(41+24)=0.63$ and $\beta_{2}=24 /(41+24)=0.37$. Now taking a VLR area composed of 7.7 hexagonal cells, with 5 location areas of 11,10 and 9 cells, the results obtained are: $\beta_{1}=34 /(34+24)=0.59$ and $\beta_{2}=24 /(34+24)=0.41$, similar to the previous case, although $\beta_{2}$ becomes noticeably larger.

\subsection{Advanced Algorithm}

Taking into account for each particular cell the exact number of neighboring cells sharing location area borders whether or not being administered by the same VLR, the number of xs and -s obtained in this way rises in comparison with the simple algorithm, but the results remain quite similar for some of the cases. In this sense, for the structure of the square cells with 5 location areas per VLR, the results obtained are: $\beta_{1}=110 /(110+80)=0.58$, and $\beta_{2}=$ $80 /(110+80)=0.42$.

For the 7.7 hexagonal cells structure, the outcome is: $\beta_{1}=84 /(84+54)=0.61$ and $\beta_{2}=54 /(84+54)=0.39$, again similar to previous results, although this time $\beta_{2}$ becomes noticeably smaller. More results obtained by means of this algorithm are presented in Table 1, and some of the geographical configurations are shown in Figure 2.

From Table 1 it can be noticed that for a same VLR administered zone dimension and cell shape, as the size of the location areas rises, $\beta_{1}$ declines and complementarily, $\beta_{2}$ grows. Regarding the number of xs, it remains constant regardless of the location areas shape and size for a fixed geographical area covered by the VLR, as this number just depends on the size and shape of that VLR area. In order to minimize the number of $x$ in proportion to $\bullet$ s, and therefore decrease the values of the $\beta_{21}$ and $\beta_{22}$ parameters, the VLR area should be as regular as possible, and containing the largest possible number of cells within (for instance, 100 hexagonal cells served by an only VLR bring $78 \mathrm{xs}$, while two groups of 49 square cells served by an VLR each, bring $108 \mathrm{xs}$ ). Furthermore, considering a VLR area of $m \cdot m$ cells, the number of xs in a square cells deployment will be $20+12 \cdot(m-2)$, while for hexagonal cells, the number of xs will be $14+8 \cdot(m-2)$, considerably lower.

The number of $\bullet$ s depends on the size and shape of the location areas. The smaller the location areas, the larger the total length of shared borders and, consequently the larger the number of $\bullet$. In the same sense, the more irregular the shape of the location areas, the larger the number of $\bullet$. Obviously, for a fixed size of location areas, the larger the geographical zone covered by the VLR, the higher the number of $\bullet$ s. In order to minimize the number of $\bullet$ s, and therefore diminish the values of the $\beta_{1}$ parameter, the shape of the location areas should be square, and their size as large as possible, ideally to fit one location area in one VLR zone. 
International Journal of Computer Networks \& Communications (IJCNC) Vol.4, No.2, March 2012

Making use of the calculated $\beta$ parameters, the location update costs for different cellular deployments and Location Management strategies will be obtained next.

Table 1. Calculation of $\beta$ parameters for different network deployments.

\begin{tabular}{|c|c|c|c|c|c|c|c|c|c|c|}
\hline $\begin{array}{c}\text { Cell } \\
\text { Shape }\end{array}$ & $\begin{array}{c}\text { VLR } \\
\text { administered } \\
\text { zone } \\
\text { dimension } \\
\end{array}$ & $\begin{array}{c}\text { Number } \\
\text { of L.A.s } \\
\text { per VLR }\end{array}$ & $\begin{array}{c}\text { Number } \\
\text { of cells } \\
\text { per L.A. }\end{array}$ & $\begin{array}{c}\text { Regularity } \\
\text { of shape of } \\
\text { L.A.S }\end{array}$ & $\begin{array}{c}\text { No. } \\
\mathbf{x}\end{array}$ & $\begin{array}{c}\text { No. } \\
\bullet\end{array}$ & $\beta_{1}$ & $\beta_{2}$ & $\beta_{21}$ & $\beta_{22}$ \\
\hline Hexagonal & 7 cells $\cdot 7$ cells & 5 & $9,10,11$ & Good & 54 & 84 & 0.61 & 0.39 & 0.312 & 0.078 \\
\hline Hexagonal & 7cells $\cdot 7$ cells & 4 & $9,12,16$ & Very good & 54 & 50 & 0.48 & 0.52 & 0.416 & 0.104 \\
\hline Hexagonal & 10cells $\cdot 10$ cells & 9 & $9,12,16$ & Very good & 78 & 144 & 0.65 & 0.35 & 0.28 & 0.07 \\
\hline Hexagonal & 10 cells $\cdot 10$ cells & 4 & 25 & Very good & 78 & 74 & 0.49 & 0.51 & 0.408 & 0.102 \\
\hline Hexagonal & 10cells $\cdot 10$ cells & 2 & 50 & Very good & 78 & 38 & 0.33 & 0.67 & 0.536 & 0.134 \\
\hline Square & 7 cells $\cdot 7$ cells & 17 & 2,3 & Good & 80 & 248 & 0.76 & 0.24 & 0.192 & 0.048 \\
\hline Square & 7 cells $\cdot 7$ cells & 16 & $1,2,4$ & Very good & 80 & 191 & 0.7 & 0.3 & 0.24 & 0.06 \\
\hline Square & 7cells $\cdot 7$ cells & 9 & $4,6,9$ & Very good & 80 & 136 & 0.63 & 0.37 & 0.296 & 0.074 \\
\hline Square & 7cells $\cdot 7$ cells & 6 & $6,8,9,12$ & Very good & 80 & 106 & 0.54 & 0.41 & 0.328 & 0.082 \\
\hline Square & 7 cells $\cdot 7$ cells & 5 & 9,10 & Medium & 80 & 110 & 0.58 & 0.42 & 0.336 & 0.084 \\
\hline Square & 7cells $\cdot 7$ cells & 4 & $9,12,16$ & Very good & 80 & 72 & 0.47 & 0.53 & 0.424 & 0.106 \\
\hline Square & 7cells $\cdot 7$ cells & 3 & $12,16,21$ & Good & 80 & 50 & 0.38 & 0.62 & 0.496 & 0.124 \\
\hline Square & 7cells $\cdot 7$ cells & 2 & 21,28 & Very good & 80 & 38 & 0.32 & 0.68 & 0.544 & 0.136 \\
\hline Square & 10cells $\cdot 10$ cells & 33 & 3,4 & Good & 116 & 550 & 0.83 & 0.17 & 0.136 & 0.034 \\
\hline Square & 10cells $\cdot 10$ cells & 16 & $4,6,9$ & Very good & 116 & 300 & 0.72 & 0.28 & 0.224 & 0.056 \\
\hline Square & 10cells $\cdot 10$ cells & 9 & $3,4,12,15$ & Good & 116 & 208 & 0.64 & 0.36 & 0.288 & 0.072 \\
\hline Square & 10cells $\cdot 10$ cells & 9 & $9,12,16$ & Very good & 116 & 208 & 0.64 & 0.36 & 0.288 & 0.072 \\
\hline Square & 10cells $\cdot 10$ cells & 4 & 25 & Very good & 116 & 108 & 0.48 & 0.52 & 0.416 & 0.104 \\
\hline Square & 10cells $\cdot 10$ cells & 3 & 30,40 & Very good & 116 & 112 & 0.49 & 0.51 & 0.408 & 0.102 \\
\hline Square & 10 cells $\cdot 10$ cells & 2 & 50 & Very good & 116 & 56 & 0.33 & 0.67 & 0.536 & 0.134 \\
\hline
\end{tabular}


International Journal of Computer Networks \& Communications (IJCNC) Vol.4, No.2, March 2012
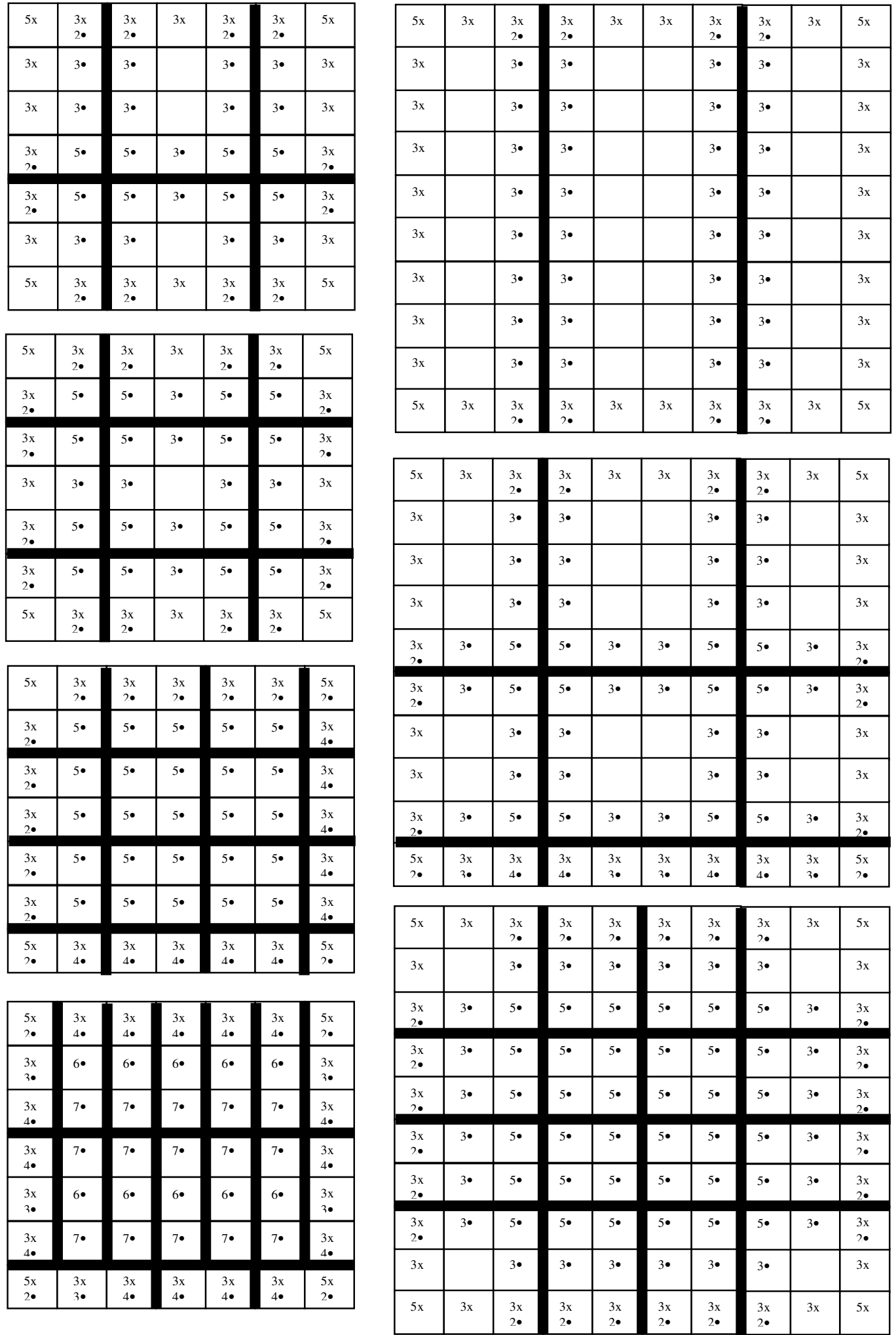

Figure 2. Examples of calculation of the $\beta$ parameters for different VLR administered zones and different location area structures for square cells. 


\section{Location UPDATE COSTS FOR THE Classical STRATEgY}

Making use of (1), we can represent the evolution of the location update costs with the number of cells per location area for the classical strategy, for different cellular structures.

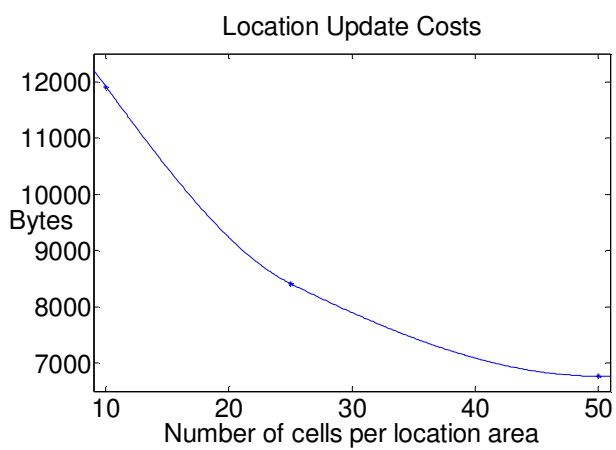

a) $10 \cdot 10$ hexagonal cells

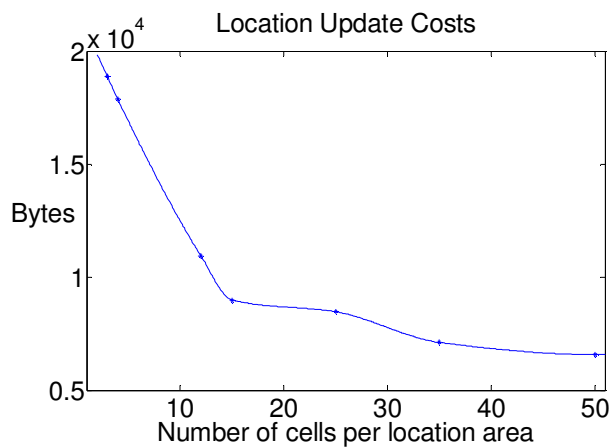

b) $10 \cdot 10$ square cells

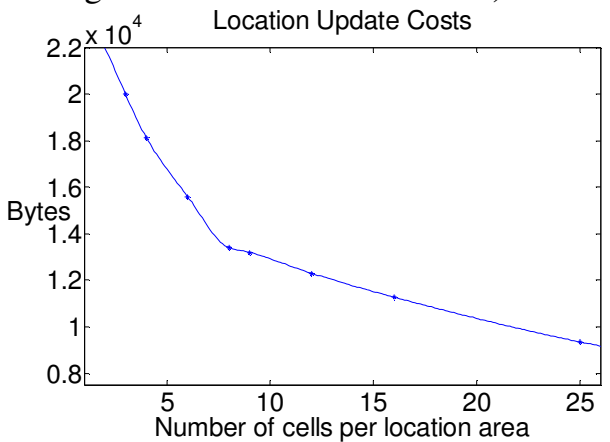

c) 7.7 square cells

Figure 3. Evolution of the location update costs with the number of cells per location area for the classical strategy, for different cellular structures.

From Figure 3, it can be observed the exponentially decreasing behavior of the location update costs with the number of cells per location area. For the same number of cells per location area, due to the dependency of the location update costs with the $\beta$ parameters, and these themselves with the particular deployment, a descent in the location update costs ranging from $5 \%$ for 3 cells per location area to $10 \%$ for 25 cells per location area is observed for larger VLR administered areas (specifically comparing the areas of 7.7 and $10 \cdot 10$ square cells). Consequently, the larger the VLR administered area, the lower the location update costs, with greater declines the larger the amount of cells in the location areas.

\section{Location Update Costs for the User Profile-Based Algorithm}

Taking five different sets of probabilities for the location areas, and considering three different schemes with 3, 5 and 9 location areas administered by the user profile-based algorithm, Figure 4 is obtained for the location update costs:

Sets of 3 Location Areas:

Probabilities: $\alpha 1=0.4, \alpha 2=0.1, \alpha 3=0.05$

Probabilities: $\alpha 1=0.5, \alpha 2=0.1, \alpha 3=0.05$

Probabilities: $\alpha 1=0.6, \alpha 2=0.1, \alpha 3=0.05$

Probabilities: $\alpha 1=0.7, \alpha 2=0.1, \alpha 3=0.05$

Probabilities: $\alpha 1=0.8, \alpha 2=0.1, \alpha 3=0.05$ 
International Journal of Computer Networks \& Communications (IJCNC) Vol.4, No.2, March 2012

Sets of 5 Location Areas:

Probabilities: $\alpha 1=0.4, \alpha 2=0.1, \alpha 3=0.05, \alpha 4=0.02, \alpha 5=0.01$

Probabilities: $\alpha 1=0.5, \alpha 2=0.1, \alpha 3=0.05, \alpha 4=0.02, \alpha 5=0.01$

Probabilities: $\alpha 1=0.6, \alpha 2=0.1, \alpha 3=0.05, \alpha 4=0.02, \alpha 5=0.01$

Probabilities: $\alpha 1=0.7, \alpha 2=0.1, \alpha 3=0.05, \alpha 4=0.02, \alpha 5=0.01$

Probabilities: $\alpha 1=0.8, \alpha 2=0.1, \alpha 3=0.05, \alpha 4=0.02, \alpha 5=0.01$

Sets of 9 Location Areas:

Probabilities: $\alpha 1=0.4, \alpha 2=0.05, \alpha 3=0.03, \alpha 4=0.02, \alpha 5=0.01, \alpha 6=0.008, \alpha 7=0.005, \alpha 8=0.003, \alpha 9=0.002$ Probabilities: $\alpha 1=0.5, \alpha 2=0.05, \alpha 3=0.03, \alpha 4=0.02, \alpha 5=0.01, \alpha 6=0.008, \alpha 7=0.005, \alpha 8=0.003, \alpha 9=0.002$ Probabilities: $\alpha 1=0.6, \alpha 2=0.05, \alpha 3=0.03, \alpha 4=0.02, \alpha 5=0.01, \alpha 6=0.008, \alpha 7=0.005, \alpha 8=0.003, \alpha 9=0.002$ Probabilities: $\alpha 1=0.7, \alpha 2=0.05, \alpha 3=0.03, \alpha 4=0.02, \alpha 5=0.01, \alpha 6=0.008, \alpha 7=0.005, \alpha 8=0.003, \alpha 9=0.002$ Probabilities: $\alpha 1=0.8, \alpha 2=0.05, \alpha 3=0.03, \alpha 4=0.02, \alpha 5=0.01, \alpha 6=0.008, \alpha 7=0.005, \alpha 8=0.003, \alpha 9=0.002$

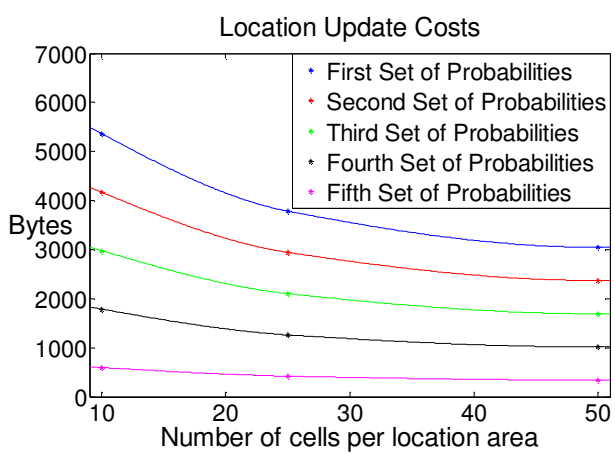

a) $10 \cdot 10$ hex. Cells

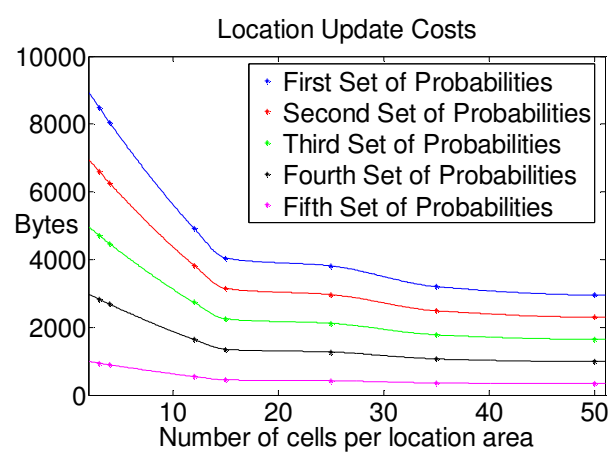

b) $10 \cdot 10$ sqr. Cells

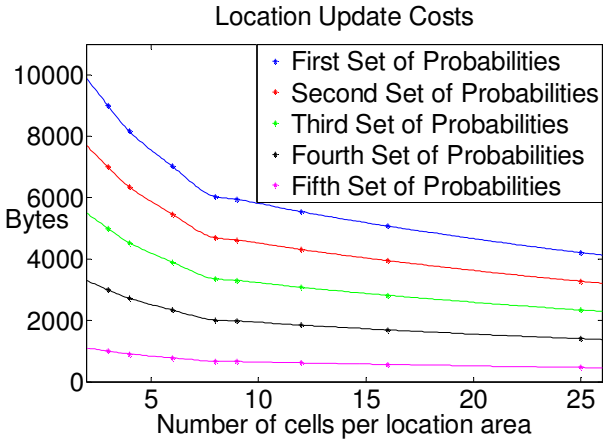

c) $7 \cdot 7$ sqr. Cells

1) 3 Location Areas administered by the user profile-based algorithm

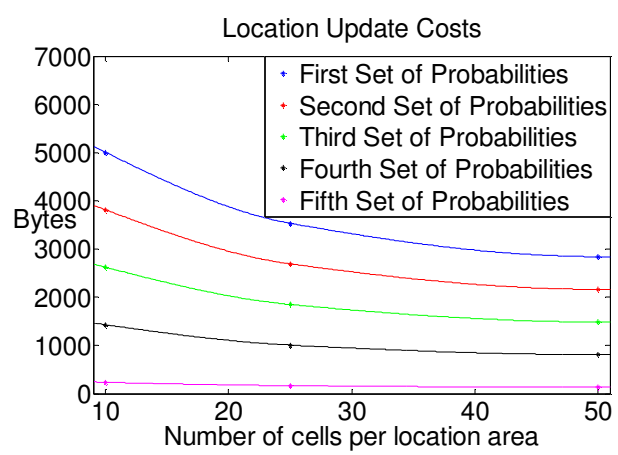

a) $10 \cdot 10$ hex. Cells

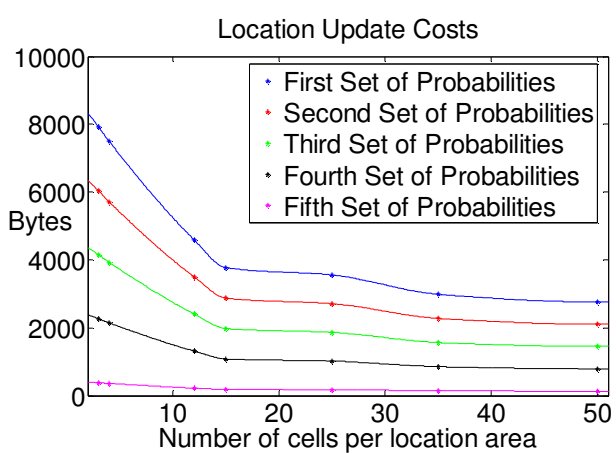

b) $10 \cdot 10$ sqr. Cells 


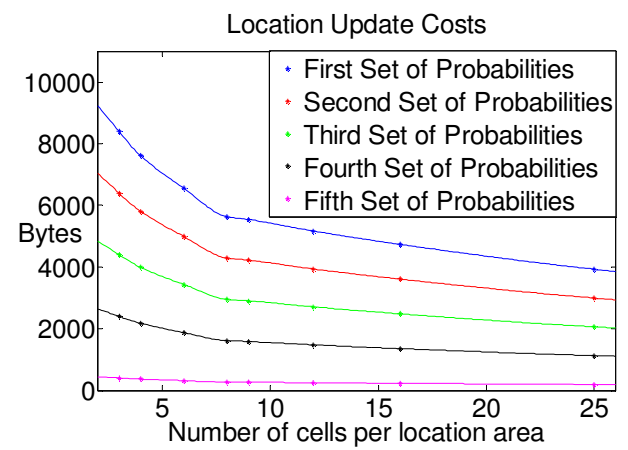

c) 7.7 sqr. Cells

2) 5 Location Areas administered by the user profile-based algorithm

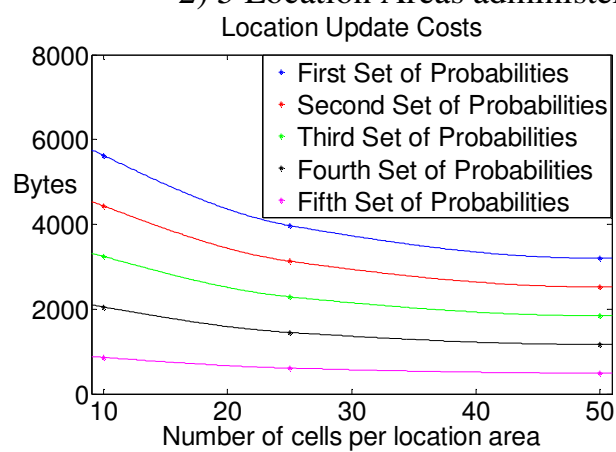

a) $10 \cdot 10$ hex. Cells

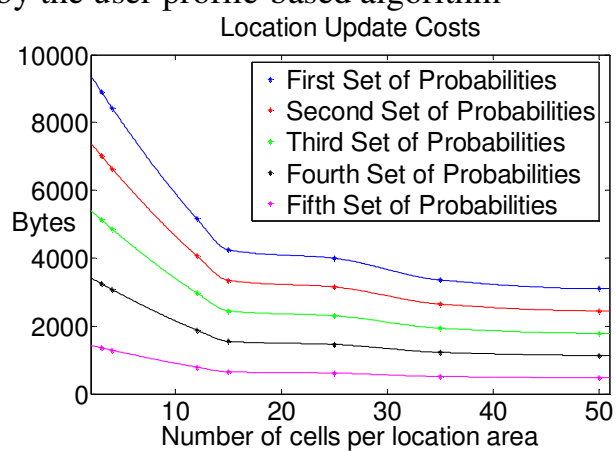

b) $10 \cdot 10$ sqr. Cells

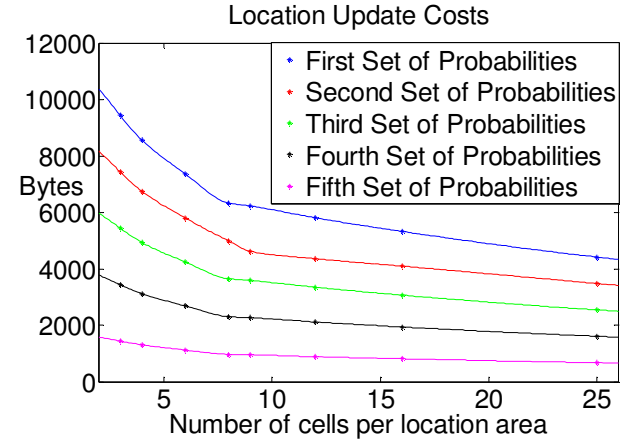

c) 7.7 sqr. Cells

3) 9 Location Areas administered by the user profile-based algorithm

Figure 4. Location update costs considering the user profile-based algorithm for different VLR administered zone sizes, and different cellular structure deployments.

Matching with expected results, the location update costs follow an exponentially decreasing behavior with the number of cells per location area. The speed of the descents depends on the deployments characteristics, and measurements show that rising from 10 to 25 the number of cells per location area, the reduction in the costs for the 7.7 square cells structure is the lowest $(35.7 \%)$ among the analyzed deployments, followed by the $10 \cdot 10$ square cells structure $(37.75 \%)$ and then the $10 \cdot 10$ hexagonal cells structure $(41.58 \%)$. However, the most important percentage falls take place for variations of lower numbers of cells per location areas and, for instance, rises from 3 to 15 in the number of cells per location area bring a reduction in the costs of $110 \%$ for the $10 \cdot 10$ square cells structure, while for the 7.7 square cells structure, this value gets below $77 \%$. Consequently, it can be concluded that the larger the VLR administered zone, the higher the decreasing speed of the location update costs with the number of cells per location area and, also, for the same size of the deployment structure, the speed of the descent is 
higher for the hexagonal cells structure than for the square cells one. The physical explanation to this statement is based on the fact that considering two VLR administered zones of different size, rises in the number of cells per location area (making the size of the location areas in the smaller zone get closer to the whole VLR administered zone itself) bring proportionally larger reductions in the number of $\bullet$ s for the small VLR area, meaning proportionally larger increases in $\beta_{2}$, which is reflected in a relatively slower descent in the costs compared to the same number of cells per location area in a larger VLR area. In other words, the smaller the VLR administered zone, the slower the decreasing speed of the location update costs with the number of cells per location area.

Among the analyzed structures, the ones with hexagonal cells bring the lowest location update costs, reasoned by the fact that making use of the advanced algorithm for the calculation of the $\beta$ parameters, the percentage reduction in the hexagonal cell structures with respect to the square cells structures is always higher for the number of xs than for the number of $\bullet$ s, as shown in Table 2.

Table 2. Comparison of the percentage reduction of xs and $\bullet$ in the advanced algorithm for the hexagonal cells with respect to the square cells.

\begin{tabular}{|c|c|c|c|c|}
\hline $\begin{array}{c}\text { VLR administered } \\
\text { zone size }\end{array}$ & $\begin{array}{c}\text { No. Location } \\
\text { Areas }\end{array}$ & $\begin{array}{c}\text { No. cells } \\
\text { per L. A. }\end{array}$ & $\begin{array}{c}\text { Percentage of } \\
\text { reduction in } \mathbf{x}\end{array}$ & $\begin{array}{c}\text { Percentage of } \\
\text { reduction in } ~\end{array}$
\end{tabular}

Therefore, the hexagonal cells structures will present relatively lower values of $\beta_{21}$ and $\beta_{22}$, which account for the highest terms in the location update costs, and consequently the costs will be lower. However, from the previous table it can be inferred that as the number of cells per location area increases, the difference in the percentage reduction between xs and $\bullet$ s tends to decline, and consequently the reduction in the location update costs will diminish.

For the square cells structure, measurements show that the VLR administered zone of size 10.10 brings lower costs than the 7.7 structure, around $5.5 \%$ for the particular case of location areas containing mainly 3 cells, regardless of the number of location areas managed in the user profile-based algorithm. This same behavior is observed for the hexagonal cells structures, and matches with the expected theoretical results taking into account the advanced algorithm, in the sense that the larger the VLR administered zone, the larger its perimeter and consequently the number of xs, but its proportional growth of surface, and therefore of the number of $\bullet$ s (if the amount of cells per location area is maintained), will be bigger. Specifically, for a square VLR zone containing $L$ cells within its side, the ratio between the area and perimeter is $L^{2} /(4 \cdot(L-1))$, which approaches to $L / 4$ for large enough zones. In conclusion, increases in the VLR administered zone (maintaining the number of cells per location area), bring lower values for the $\beta_{2}$ parameter and consequently lower location update costs.

Regarding the amount of location areas controlled by the user profile-based algorithm, the larger the summation of their probabilities, the lower the location update costs, regardless of the actual number of those location areas. 
Comparing different sets of location areas managed by the user profile-based algorithm, the percentage reductions in the location update costs due to higher summations of the probabilities of the location areas (considering schemes with similar probability summations) tend to grow when those summations approach unity. In other words, the lower the location areas probability summations, the smaller the location update costs percentage variations compared with schemes of similar summations. For instance, considering the structures previously analyzed, when the probability summations are 0.55 for the 3 location areas scheme, 0.58 for the 5 one, and 0.528 for the 9 one, the location update costs reduction percentage of the 3 location areas scheme with respect to the 9 one is $4.66 \%$, and the reduction from the 5 one with respect to the 3 one is $6.6 \%$. On the other hand, for the same cellular structures but with probability summations respectively of $0.95,0.98$ and 0.928 , the percentage costs reductions are now respectively $30.55 \%$ and $60 \%$, which are much higher than before.

Calculating the ratio between the user profile-based algorithm and the classical strategy for the location update costs, in terms of the mobility predictability level, defined as $\sum_{i=1}^{k} \frac{\alpha_{i}}{i}$, with $k$ being the number of location areas managed by the user profile-based algorithm, the results in Figure 5 are obtained.

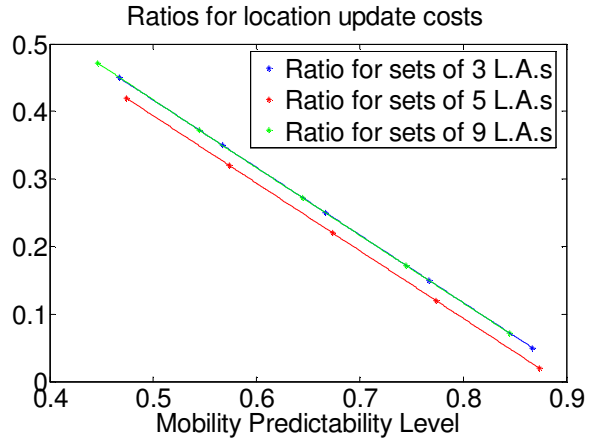

a) Ratios

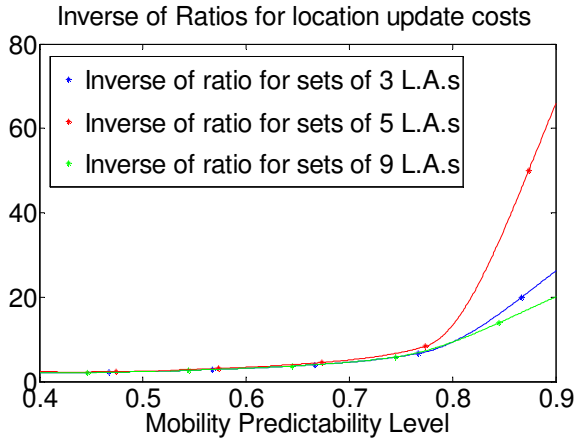

b) Inverse of ratios

Figure 5. Comparison between the user profile-based algorithm and the classical strategy for location update costs in terms of the mobility predictability level.

From Figure 5, it can be observed that the larger the predictability of the mobile terminal being tracked down by the user profile-based algorithm, the smaller the ratios in the location update costs with respect to the classical strategy. It should be noticed that when the predictability approaches unity, the inverse of the ratio can reach values above 50, which means an excellent performance for the user profile-based algorithm in terms of location update costs savings.

\section{Conclusions}

In this paper, we have analyzed the location update signaling costs for user profile-based algorithms, and we have presented new methods to obtain the $\beta$ parameters (useful in the calculation of the location update costs for different Location Management strategies). From these results, practical guidelines can be obtained for the networks designers in order to minimize signaling costs. In particular, the minimization of the $\beta_{1}$ parameter is achieved through enlargements in the location area size, ideally with square shape and fitting in the surface of a VLR administered zone. The minimization of the $\beta_{21}$ and $\beta_{22}$ parameters requires reductions in the size of the location areas and rises in the number of cells within the VLR administered zone, whose shape should be as regular as possible. 
International Journal of Computer Networks \& Communications (IJCNC) Vol.4, No.2, March 2012

And from the analysis of the location update costs for the user profile-based algorithm, we can draw the following conclusions:

Increases in the VLR administered zone size (keeping the number of cells per location area fixed), bring declines in the location update costs and rises in their decreasing speed with the number of cells per location area.

Hexagonal cells schemes deliver lower location update costs and higher decreasing speeds in those costs than the square ones, although the difference is reduced as the number of cells per location area grows.

$>$ The larger the summation of the probabilities of the location areas controlled by the user profile-based algorithm, the lower the location update costs, regardless of the actual number of those location areas.

In comparison with the classical strategy, the more foreseeable the behavior of the mobile terminal being tracked down by the user profile-based algorithm, the lower the location update costs ratio between the latter and the former strategies.

\section{REFERENCES}

[1] Vijayakumar, H.; Ravichandran, M.; "Efficient location management of mobile node in wireless mobile ad-hoc network", Proceedings of National Conference on Innovations in Emerging Technology, NCOIET'11, p 77-84, 2011

[2] Biswash, Sanjay Kumar; Kumar, Chiranjeev; "Modelling and cost analysis of location management scheme for PCS networks", International Journal of Information and Communication Technology, v 3, n 1, p 53-67, April 2011

[3] Yu, F. Richard; Wong, Vincent W.S.; Song, Joo-Han; Leung, Victor C.M.; Chan, Henry C.B.; "Next generation mobility management: An introduction", Wireless Communications and Mobile Computing, v 11, n 4, p 446-458, April 2011.

[4] Chung, Yun Won; "A multi tier group location management scheme for multi interface mobile station", 4th IFIP International Conference on New Technologies, Mobility and Security, NTMS 2011

[5] Chung, Yun Won; "A novel group location management scheme based on route information of public transportation system", IEICE Transactions on Communications, v E94-B, n 2, p 477 483, February 2011

[6] Almeida-Luz, Sónia M.; Vega-Rodríguez, Miguel A.; Gómez-Púlido, Juan A.; Sánchez-Pérez, Juan M.; "Differential evolution for solving the mobile location management", Applied Soft Computing Journal, v 11, n 1, p 410-427, January 2011

[7] Gállego, J.; Canales, M.; Hernández-Solana, Á.; Valdovinos, A.; “Adaptive paging schemes for group calls in mobile broadband cellular systems", IEEE International Symposium on Personal, Indoor and Mobile Radio Communications, PIMRC, p 2444-2449, 2010.

[8] Martin, E.; "Multimode radio fingerprinting for localization", IEEE Radio and Wireless Week, IEEE Topical Conference on Wireless Sensors and Sensor Networks, Phoenix, Arizona, pp. 383386, 2011.

[9] Goel, Ashish; Gupta, Navankur; Kumar, Prakhar; "A speed based adaptive algorithm for reducing paging cost in cellular networks", Proceedings - 2009 2nd IEEE International Conference on Computer Science and Information Technology, ICCSIT 2009, p 22-25, 2009.

[10] Casares-Giner, Vicente; García-Escalle, Pablo; “A lookahead strategy for movement-based location update in wireless cellular networks", ITNG 2009 - 6th International Conference on Information Technology: New Generations, p 1171-1177, 2009. 
International Journal of Computer Networks \& Communications (IJCNC) Vol.4, No.2, March 2012

[11] Martin, E.; Lin, G.; Weber, Matt; Pesti, Peter; Woodward, M.; "Unified analytical models for location management costs and optimum design of location areas", 5th International Conference on Collaborative Computing: Networking, Applications and Worksharing, CollaborateCom, 2009, pp. 1-10.

[12] Tabbane, S., "An alternative strategy for location tracking" IEEE Journal on Selected Areas in Communications, v.13, n.5, pp. 880-892, 1995.

[13] Tabbane, S., "Comparison between the alternative location strategy (AS) and the classical location strategy (CS)" WINLAB, Rutgers Univ., Piscataway, NJ, Technical Report 37, Sept. 1992.

[14] Pollini, G., and Tabbane, S., "The intelligent network signalling and switching costs of an alternate location strategy using memory" Proceedings IEEE, Vehicular Technology Conference, Secaucus, NJ, pp. 931-934, May 18-20, 1993.

[15] Amar Pratap Singh, J.; "Intelligent location management using soft computing technique", 2nd International Conference on Communication Software and Networks, pp. 343-346, 2010.

[16] Mokbel, Mohamed F.; Levandoski, Justin J.; "Toward context and preference-aware locationbased services", Proceedings of the 8th ACM International Workshop on Data Engineering for Wireless and Mobile Access in Conjunction with ACM SIGMOD / PODS, pp. 25-32, 2009.

[17] Koutrika, Georgia; Ioannidis, Yannis; "Constrained optimalities in query personalization", Proceedings of the ACM SIGMOD International Conference on Management of Data, pp. 73-84, 2005.

[18] Koutrika, Georgia; Ioannidis, Yannis; "Personalization of queries in database systems", Proceedings - International Conference on Data Engineering, v 20, pp. 597-608, 2004.

[19] Koutrika, Georgia; Loannidis, Yannis; "Personalized queries under a generalized preference model”, Proceedings - International Conference on Data Engineering, pp. 841-852, 2005.

[20] Stefanidis, Kostas; Pitoura, Evaggelia; "Fast contextual preference scoring of database tuples", Advances in Database Technology - EDBT 2008 - 11th International Conference on Extending Database Technology, Proceedings, pp. 344-355, 2008.

[21] Stefanidis, Kostas; Pitoura, Evaggelia; Vassiliadis, Panos; "Adding context to preferences", Proceedings - International Conference on Data Engineering, pp. 846-855, 2007.

[22] Pollini, G., "Capacity of an IEEE 802.6 based cellular packet switch” Proceedings IEEE ICC 93, Geneva, Switzerland, pp. 1264-1268, May 1993.

[23] Xiao, Y., "Optimal location management for two-tier PCS networks", Computer Communications, v 26, n 10, pp. 1047-1055, 2003.

[24] Park, J., Choi, J., Choi, M., "A dynamic location update scheme based on the number of movements and the movement history for personal communications networks", IEICE Transactions on Communications, v E85-B, n 10, pp. 2300-2310, 2002.

[25] Lin, H., Lee, S., "A Track-Presetting strategy in PCS using hierarchical location databases" Computer Communications, v 25, n 18, p 1727-1735, 2002.

[26] Kong, K., Gil, J., Han, Y., Song, U., Hwang, C., “A forwarding pointer-based cache scheme for reducing location management cost in PCS networks", Journal of Information Science and Engineering, v 18, n 6, p 1011-1025, 2002.

[27] Lo, S., Chen, A., "Adaptive region-based location management for PCS systems", IEEE Transactions on Vehicular Technology, v 51, n 4, p 667-676, 2002.

[28] Mao, Z.; Douligeris, C., "Location-based mobility tracking scheme for PCS networks", Computer Communications, v 23, n 18, p 1729-1739, 2000.

[29] Pollini, G., and Gitlin, R., "Optimum location area sizes and reverse virtual call setup in PCS networks" Proceedings IEEE Vehicular Technology Conference 95, Chicago, IL, p. 140-144, July 1995. 
International Journal of Computer Networks \& Communications (IJCNC) Vol.4, No.2, March 2012

[30] CCIR "Future public land mobile telecommunications systems” Doc. 8/1014-E, December 1989.

[31] Yuen, W., Wong, W., "Dynamic location area assignment algorithm for mobile cellular systems", IEEE International Conference on Communications, v 3, pp. 1385-1389, 1998.

[32] Lei, Z., Rose, C., "Probability criterion based location tracking approach for mobility management of personal communications systems", Conference Record / IEEE Global Telecommunications Conference, v 2, p 977-981, 1997.

[33] Chang, R., Chen, K., "Dynamic mobility tracking for wireless personal communication networks", Annual International Conference on Universal Personal Communications - Record, 1997, v 2, pp. 448-452, 1997.

[34] Watanabe, Y., Yabusaki, M., "Mobility/traffic adaptive location management", IEEE Vehicular Technology Conference, v 56, n 2, pp. 1011-1015, 2002.

[35] Cao, P., Wang, X., Huang, Z., "Dynamic location management scheme based on movementstate", Acta Electronica Sinica, v 30, n 7, p 1038-1040, 2002.

[36] Tabbane, S., "Location management methods for third-generation mobile systems", IEEE Communications Magazine, pp. 72-84, August 1997.

[37] D. MacFarlane and S. Griffin, "The MPLA Vision of UMTS", CODIT/BT/PM-005/1.0, Feb.1994.

\section{Authors}

E. Martin is carrying out research in the Department of Electrical Engineering and Computer Science at University of California, Berkeley. He holds a MS in Telecommunications Engineering from Spain and a $\mathrm{PhD}$ from England within the field of location management for mobile telecommunications networks. He has research experience in both industry and academia across Europe and USA, focusing on wireless communications, sensor networks, signal processing and localization.

R. Bajesy received the Master's and Ph.D. degrees in electrical engineering from the Slovak Republic, and the Ph.D. in computer science from Stanford University, California. She is a Professor of Electrical Engineering and Computer Sciences at the University of California, Berkeley. Prior to joining Berkeley, she headed the Computer and Information Science and Engineering Directorate at the National Science Foundation. Dr. Bajcsy is a member of the National Academy of Engineering and the National Academy of Science Institute of Medicine as well as a Fellow of the Association for Computing Machinery (ACM) and the American Association for Artificial Intelligence. In 2001, she received the ACM/Association for the Advancement of Artificial Intelligence Allen Newell Award, and was named as one of the 50 most important women in science in the November 2002 issue of Discover Magazine. 